\title{
Prospecção de zonas potenciais para manejo diferenciado em agricultura de precisão utilizando-se padrões de solo-planta-clima ${ }^{1}$
}

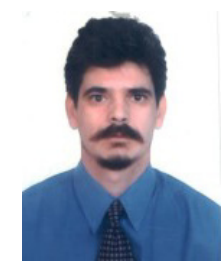

\author{
Antonio O. Santos ${ }^{2}$, Wander J. Pallone Filho ${ }^{3}$, Maria R. G. Ungaro ${ }^{4}$, Antonio C. L. Lino ${ }^{2}$ \& Bernadete Radin ${ }^{5}$ \\ Apoio: Fundação de Apoio à Pesquisa do Estado de São Paulo - FAPESP \\ 2 IAC/APTA. CP 26, CEP 13201-970, Jundiaí, SP. E-mail: odairsan@iac.sp.gov.br (Foto) e lino@iac.sp.gov.br \\ 3 UNICAMP/FEAGRI, CP 6011, CEP 13083-970, Campinas, SP. E-mail: wander@cati.sp.gov.br \\ ${ }^{4}$ IAC/APTA. CP 28, CEP 12001-970, Campinas, SP. Fone: (19) 3231-5422. E-mail: ungaro@iac.sp.gov.br \\ ${ }^{5}$ FEPAGRO. Rua Gonçalves Dias 570, CEP 90130-060, Porto Alegre, RS. E-mail: radin@fepagro.rs.gov.br
}

Protocolo 2 - 3/1/2003 - Aprovado em 8/10/2003

\begin{abstract}
Resumo: A agricultura de precisão contempla a aplicação da informação tecnológica aos processos produtivos. A implementação de programas nesta linha depende da integração de dados de solo, planta e clima, para a geração de informações úteis. O reconhecimento de padrões em propriedades de solo e planta, é fundamental para a viabilização de práticas nessa área, principalmente para uma abordagem de lavouras por zonas ou classes de manejo. Visando estudar aspectos do delineamento de zonas potenciais para manejo diferenciado e distribuição espacial do potencial produtivo, conduziu-se um experimento na região de Angatuba, SP, Brasil. Uma amostragem intensiva de variáveis de solo e planta, além de dados do microclima, foi realizada em uma área de 40 ha cultivados com milho-sequeiro, em duas safras seguidas, além da integração dessas informações para o reconhecimento de padrões de solo e definição de zonas potenciais de manejo. Também se fez a verificação conjunta com os padrões de distribuição da malha de produtividade, durante duas estações de crescimento de milho-sequeiro. O processo de segmentação utilizado mostrou-se adequado para o delineamento proposto, ao mesmo tempo em que foi observado que a definição de zonas com potencial para manejo diferenciado é um processo dinâmico, podendo ser influenciado por propriedades do meio e condições climáticas.
\end{abstract}

Palavras-chave: milho, gestão agrícola, análise de “agrupamentos", SIG

\section{Exploratory analysis of potential zones for differential management in precision agriculture using soil-plant-climate patterns}

\begin{abstract}
Crop site-specific management, also known as precision farming, is related to the application of information technology to the agricultural systems. The implementation of precision farming programs depends on the integration of soil, plant and climate data in order to generate useful information. The recognition of patterns for soil and plant properties is important especially for crop zone management strategies. Aiming to study management aspects by zone delineation and spatial yield patterns, a field experiment was carried out in Angatuba, SP $\left(23^{\circ} 33^{\prime} S^{\prime} ; 48^{\circ} 18^{\prime}\right.$ W; $670 \mathrm{~m}$ ). Grid soil sampling was performed and plant and climatic data were collected in a 40 ha area cultivated with maize autumn sowing, in two consecutive growing seasons. Integration of these data was done by a procedure for soil pattern recognition, aiming a management by zone delineation and yield spatial distribution verification. The proposed integrated procedures for area segmentation showed to be promising and the management by zone delineation process suggests itself to be influenced by mean properties and climatic conditions.
\end{abstract}

Key words: maize, precision farming, cluster analysis, GIS

\section{INTRODUÇÃO}

Vários estudos recentes têm aventado a possibilidade de se adotar manejo "não homogêneo" para algumas atividades e etapas do agronegócio (Schmidt et al., 2000). De fato, um conjunto de técnicas caracterizadas como "agricultura de precisão", tem sido discutidas como alternativa para o manejo agrícola. Este conjunto se baseia no detalhamento da informação em agrossistemas, obtido através da aplicação de processos de monitoramento georreferenciado e integração de variáveis de solo, planta e clima (Stewart \& McBratney, 2000; Plant, 2001). Agricultura de precisão prevê o aproveitamento da estrutura da variação espacial de elementos do meio físico, condicionantes da produtividade agrícola, para 
avançar na racionalização da aplicação de insumos e abrandamento de eventuais impactos ambientais advindos de superdosagens. A temática tem sido discutida para as condições agrícolas do Brasil e tem havido aumento na geração da informação básica sobre a questão (p.e. Santos et al., 2001a).

As culturas graníferas, como o milho, apresentam potencial significativo para a aplicação das técnicas citadas, pela grande área de cultivo estabelecida e pela necessidade constante do aprimoramento de estratégias de manejo, na busca da sustentabilidade. Em condições de sequeiro, a exposição ao balanço hídrico circunstancial tende a contribuir para uma definição mais consistente de zonas de manejo, sob o ponto de vista do nível de contraste físico inter-zonal necessário para haver retorno econômico na implementação.

A aplicação de manejo diferenciado pelo aproveitamento de contrastes do meio físico em campos de cultivo, depende do reconhecimento de padrões de variação em propriedades de solo e de planta. Esses padrões poderiam ser utilizados tanto para relacionar causa e efeito entre fatores limitantes e produtividade quanto para fazer o delineamento e análise de subáreas de atuação, nas quais, teoricamente, se poderia manejar a aplicação de insumos a taxas constantes. A recomendação baseada no potencial produtivo de cada subárea facilitaria o manejo de recursos da produção.

Neste sentido, Fleming et al. (2000), dentre outros, correlacionaram parâmetros de solo com produção, utilizando zonas de manejo diferenciado delineadas com correlações entre sondagens de solo tradicionais e com estudos de sobreposição de mapas.

Pelo fato de projetos em agricultura de precisão produzirem grande quantidade de dados de solo e de planta, faz-se necessário utilizar-se processos ou técnicas que integrem esses dados com a finalidade de dimensionar corretamente as zonas de manejo diferenciado e, também, estudar a sua dinâmica frente às condições variáveis de manejo e clima.

Modelos "Fuzzy" têm sido utilizados para sistemas de controle. Algoritmos de grupamentos "fuzzy" são ferramentas matemáticas utilizadas para detectar similaridade entre membros de uma coleção de objetos (Windham, 1982; Ateca et al., 2001). Fridgen et al. (2000) delinearam zonas de manejo utilizando a classificação algorítmica não supervisionada "Fuzzy K-means", em dados de condutividade elétrica aparente, elevação e declividade de dois solos. O número de zonas apropriadas em cada solo foi determinado levando-se em conta a redução na variância da produção de grãos e as medidas de desempenho dos agrupamentos ("clusters"). Análise de componentes principais foi utilizada para examinar a variação das propriedades físicas do solo e parâmetros topográficos de cada zona de manejo específico. Os autores encontraram que a análise dos componentes principais e o exame da redução da variância devido à subdivisão em número crescente de zonas, podem fornecer o discernimento para a singularidade das classes criadas com o algoritmo da classificação não supervisionada.

O presente trabalho objetivou testar, através da integração das informações de solo e planta, um conjunto de procedimentos para o reconhecimento de padrões de solo, visando ao delineamento de zonas potenciais de manejo e a verificação conjunta com os padrões de distribuição da malha de produtividade de milho-sequeiro.

\section{MATERIAL E MÉTODOS}

\section{Local, época e cultivar}

$\mathrm{O}$ experimento foi conduzido na região de Angatuba, SP,

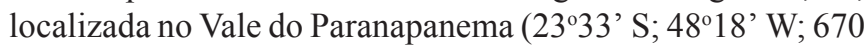
$\mathrm{m})$, em uma área de 40 ha de milho, durante as estações de crescimento de 1999/2000 e 2000/2001, com semeadura na segunda quinzena de setembro. A cultivar de milho híbrido Pioneer 30F33 foi utilizada nos dois anos experimentais, observando-se a recomendação da pesquisa regional para alto rendimento sob condições de sequeiro (Raij et al., 1997).

\section{Levantamento de dados de solo e clima}

Uma malha quadrada com 161 pontos eqüidistantes $50 \mathrm{~m}$ foi demarcada ao longo da área experimental, para levantamento de variáveis de solo, na profundidade de 0 a $20 \mathrm{~cm}$. No total, foram extraídas 322 subamostras (duas amostras por ponto) para as quais se determinaram, em laboratório, os valores de argila, silte, areias (muito grossa, grossa, média, fina e muito fina), matéria orgânica, densidade do solo, água retida a $33 \mathrm{kPa}$ e a $1500 \mathrm{kPa}$ (Camargo et al., 1986). As variáveis de solo foram estudadas em algoritmo específico para segmentação experimental da área de cultivo de milho em zonas potenciais de manejo. Um sistema de informação geográfica (SIG) foi utilizado como elemento de integração gráfico-numérica (Eastman, 1995). Para a ligação dos dados advindos da análise de segmentação e o SIG utilizado, uma interface foi construída, o que permitiu alinhar os dados em termos de formatação (Figura 1). Dados meteorológicos (precipitação pluvial, temperatura do ar, radiação solar e velocidade média do vento) foram coletados na área experimental, em estação meteorológica automática.

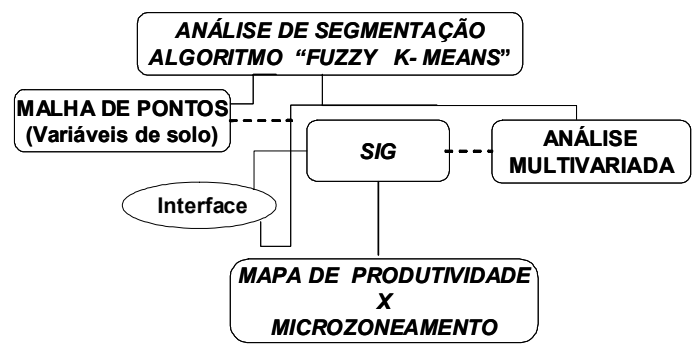

Figura 1. Diagrama geral da integração de informações em experimento com milho-sequeiro

\section{Mapeamento da produtividade do milho}

Uma malha de dados de produtividade foi obtida na área experimental nas duas safras consecutivas. Os dados foram levantados através da instrumentação de colhedora automotriz para o georreferenciamento no solo e estimativa dos dados da produtividade do milho (Santos et al., 2001a). Após a filtragem dos dados foram selecionados em torno de 16.000 pontos de produtividade em cada ano, os quais foram utilizados em análise conjunta com os dados de solo, visando ao uso de um dado independente e com significado biofísico para verificação da segmentação (microzoneamento) da área de cultivo. 


\section{Procedimento para análise de segmentação}

Para os dados de solo, um procedimento baseado em análise de segmentação multivariada (Kachigan, 1991) foi testado sobre os pontos da malha amostral e o estabelecimento da similaridade intergrupal foi efetuado. A dimensão da similaridade e a formação de subgrupos ("clusters") foram modeladas com base em procedimento “fuzzy k-means” (Bezdek, 1981). O mesmo procedimento foi utilizado por McBratney \& Odeh (1985) para classificação climática e de solo. O algoritmo "Fuzzy k-means" testa subagrupamentos de dados, buscando a minimização da variância intraclasse e a maximização da variância interclasse, fazendo a minimização da função objetivo $\mathrm{J}_{\mathrm{m}}$, com respeito a uma função característica $\mu_{\mathrm{ij}}\left(\mu_{\mathrm{ij}} \in[0,1]\right)$ e um centro de classe $\mathrm{V}_{\mathrm{i}}$ (Bezdek, 1981; McBratney \& Odeh,1985):

$$
\mathrm{J}_{\mathrm{m}}=\sum_{\mathrm{i}=1}^{\mathrm{c}} \sum_{\mathrm{j}=1}^{\mathrm{n}}\left(\mu_{\mathrm{ij}}\right)^{\mathrm{m}} \mathrm{d}^{2}\left(\mathrm{X}_{\mathrm{j}}, \mathrm{V}_{\mathrm{i}}\right)
$$

sendo:

$$
d^{2}=\left(X_{i}, V_{i}\right)=\left(X_{j}-V_{i}\right)^{T} A\left(X_{j}-V_{i}\right)
$$

donde: A é a matriz positiva ( $\mathrm{p}$ x p), sendo $\mathrm{p}$ a dimensão de cada vetor (variável de solo) $\mathrm{X}_{\mathrm{j}}(\mathrm{j}=1,2 \ldots \mathrm{n})$, c é o número de classes ("clusters"), n é o número de vetores e m é um índice do grau "fuzzy" para a classificação (Xie \& Beni, 1991). Na prática, o algoritmo é executado iterativamente, do seguinte modo:

a) Inicialização da função característica $i_{\mathrm{ij}}$ da variável de solo $\mathrm{X}_{\mathrm{j}}$ pertencente ao centro da classe $\mathrm{i}$, de modo que:

$$
\sum_{i=1}^{c} \mu_{i j}=1
$$

b) Cômputo do centro de classe $V_{i}$, para $i=1,2, \ldots$, usando-se:

$$
\mathrm{V}_{\mathrm{i}}=\frac{\sum_{\mathrm{j}=1}^{\mathrm{n}}\left(\mu_{\mathrm{ij}}\right)^{\mathrm{m}} \mathrm{X}_{\mathrm{j}}}{\sum_{\mathrm{j}=1}^{\mathrm{n}}\left(\mu_{\mathrm{ij}}\right)^{\mathrm{m}}}
$$

c) Atualização de $\grave{i}_{\mathrm{ij}}$ para cada classe, empregando-se:

$$
\mu_{i j}=\frac{\left(\frac{1}{d^{2}\left(X_{j}, V_{i}\right)}\right)^{\frac{1}{(m-1)}}}{\sum_{i=1}^{c}\left(\frac{1}{d^{2}\left(X_{j}, V_{i}\right)}\right)^{\frac{1}{(m-1)}}}
$$

Os passos b e c são repetidos até que o valor da função $\mathrm{J}_{\mathrm{m}}$ não mais diminua. O melhor resultado prático da classificação "fuzzy" é obtido na aplicação direta sobre um plano interpolado, caso em que a estimativa de transição entre classes se dá de modo gradual, mais próximo da realidade da transição entre limites de valores para variáveis de solo ligadas ao manejo do milho. O procedimento citado oferece oportunidade para utilização de "hard-clusters" e "fuzzy-clusters". Neste trabalho, os pontos da malha amostral de solo foram classificados com base em "hard-clusters" não hierárquicos, e na transferência de aporte técnico, a partir do Centro Australiano de Agricultura de Precisão (Minasny \& McBratney, 2002)

Após a segmentação da área em classes, uma análise multivariada discriminante (Grimm \& Yarnold, 2000) foi efetuada para se verificar as variáveis de solo mais importantes na separação das classes.

A expressão do potencial produtivo é um dado com significado biofísico, resultante da variação de propriedades do meio. Os resultados do mapeamento da produtividade do milho em cada ano, foram utilizados como variável independente para analisar a adequação do número de classes (zonas potenciais de manejo) em função do padrão da variância nos dados de produtividade, das condições climáticas e de manejo anual. Considerou-se que a divisão de um mapa em classes de produtividade tem um valor máximo da variância nos dados, obtido com uma única classe, ou seja, a área total. Para uma divisão crescente ocorre a divisão da variância em inter e intraclasses, sendo que um mínimo valor para a variância intraclasse é obtido com a divisão do número máximo possível de classes, que seria o próprio valor do pixel, no mapa. Neste caso, o valor atingido seria zero, pois um pixel (célula) apresenta somente um dado de produtividade. Portanto, convencionouse que o máximo benefício com a divisão da área em classes (zonas) é obtido quando ocorre relativa estabilização da variância.

A variância ponderada para cada classe $\left(\mathrm{S}_{\mathrm{z}}^{2}\right)$ em cada ano, foi calculada do seguinte modo:

$$
\mathrm{S}_{\mathrm{z}}{ }^{2}=\frac{1}{\mathrm{n}} \sum_{\mathrm{i}=1}^{\mathrm{n}}\left(\mathrm{P}_{\mathrm{i}}-\mathrm{P}_{\mathrm{m}}\right)^{2}(\mathrm{n} / \mathrm{N})
$$

donde: $\mathrm{P}_{\mathrm{i}}$ é a produtividade no pixel $\mathrm{i}, \mathrm{P}_{\mathrm{m}}$ a produtividade média em cada classe, n o número de pixels componentes da classe e $\mathrm{N}$ o número total de pixel na imagem de produtividade.

A estabilização da variância média ponderada foi utilizada como um indicador do número adequado de classes, para a segmentação da área experimental. Uma vez que grande parte da variabilidade nos dados de produtividade não pode ser explicada ou não tem significado prático, um decréscimo em $50 \%$ da variância total foi adotado como patamar mínimo para a verificação do benefício obtido na estabilização da variância dentro das classes, a partir da divisão crescente em classes. Neste sentido, a variância média ponderada, intraclasse, foi calculada do seguinte modo:

$$
\overline{\mathrm{S}}_{\mathrm{P}}^{2}=\left(\mathrm{S}_{1}^{2}+\mathrm{S}_{2}^{2}+\mathrm{S}_{\mathrm{n}}^{2}\right) / \mathrm{n}_{\mathrm{c}}
$$

em que: $n_{c}$ é o número de classes. Assim, para cada divisão de classes calculou-se a variância média ponderada intraclasse, a qual foi a seguir comparada com a variância total obtida para a classe única (mapa inteiro). 


\section{RESULTADOS E DISCUSSÃO}

Na Figura 2 estão os resultados obtidos com o agrupamento de pontos amostrais, obedecendo ao grau de similaridade entre pontos, os quais não foram interpolados para melhor clareza na demonstração. Em cada ponto foram considerados 11 atributos físicos do solo (Tabela 1). Os grupos ficaram mais bem delineados quando foram consideradas somente duas classes. A classificação a partir de três subgrupos sofreu um confundimento mais acentuado entre pontos, que se alinharam em zonas menos distintas, resultado da característica da distribuição espacial dos dados utilizados como variáveis descritoras dos pontos, que apresentaram alta variabilidade aleatória, ou seja, fraca
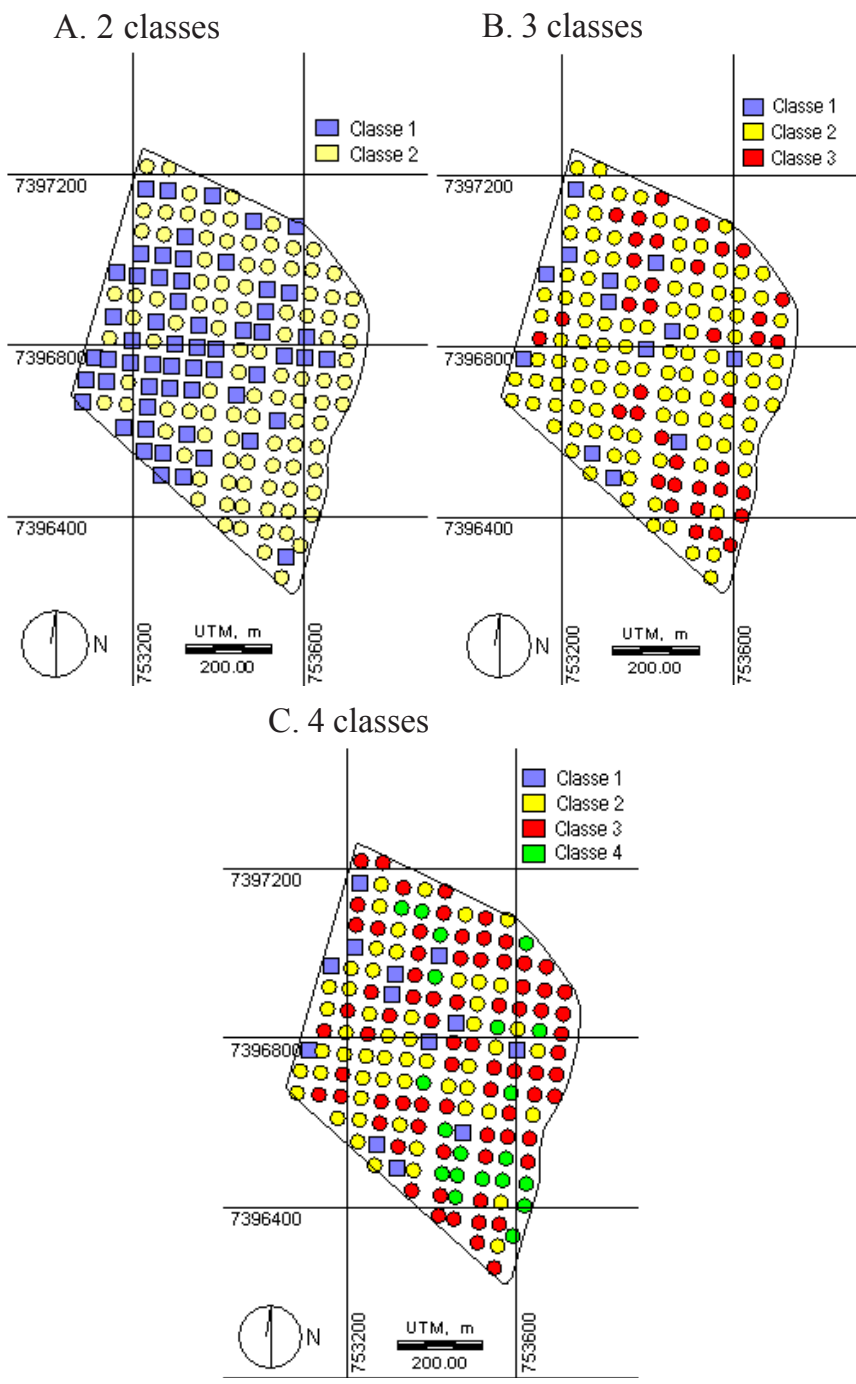

Figura 2. Resultados do agrupamento de 161 pontos para 2, 3 e 4 classes, em área de 40 ha cultivada com milho, formadas a partir do cotejo de 11 variáveis de solo por ponto amostral dependência espacial, além de alto valor de pepita (Santos et al., 2001b). Na prática, isto significa que variáveis de solo muito próximas, podem ser muito diferentes em valores.

Tabela 1. Estatística descritiva das variáveis de solo estudadas na área experimental (161 pontos)

\begin{tabular}{lrcrr}
\hline \multicolumn{1}{c}{ Atributos } & Média & $\begin{array}{c}\text { Desvio } \\
\text { Padrão }\end{array}$ & Mínimo & Máximo \\
\hline Argila & 65,35 & 6,14 & 47,60 & 83,85 \\
Silte & 24,64 & 5,92 & 2,54 & 40,50 \\
Areia muito grossa & 2,04 & 0,46 & 0,99 & 3,67 \\
Areia grossa & 1,64 & 0,49 & 0,35 & 4,60 \\
Areia média & 1,14 & 0,36 & 0,49 & 2,17 \\
Areia fina & 0,62 & 0,21 & 0,35 & 1,25 \\
Areia muito fina & 4,45 & 1,08 & 1,78 & 10,40 \\
Densidade do solo, $\mathrm{kg} \mathrm{dm}^{-3}$ & 1,33 & 0,05 & 1,17 & 143 \\
Matéria orgânica, g dm & 32,22 & 2,72 & 23,00 & 41,00 \\
Umidade (33 kPa), $\mathrm{cm}^{3} \mathrm{~cm}^{-3}$ & 0,46 & 0,017 & 0,39 & 0,49 \\
Umidade (1500 kPa), $\mathrm{cm}^{3} \mathrm{~cm}^{-3}$ & 0,26 & 0,04 & 0,24 & 0,27 \\
\hline
\end{tabular}

O procedimento usado para segmentação permite a utilização de outras variáveis, como de fertilidade do solo e de topografia; além disso, a inclusão de variáveis mais elaboradas para descrição da dinâmica da água no solo, como resistência à penetração de raízes e taxa de oxigenação das raízes, poderia contribuir para uma correlação maior entre dados de solo e de planta, em estudos desta natureza, porém algumas dessas variáveis são difíceis e caras de ser obtidas, o que inviabiliza o seu uso para grandes áreas. O levantamento de dados, principalmente de solo, poderia ser implementado com o uso de novas tecnologias, como a videografia multiespectral, sobretudo para áreas com maior extensão de cultivo, nas quais é preciso considerar os aspectos técnicos e econômicos que viabilizem o levantamento de dados.

A análise discriminante evidenciou, como mais importantes na separação das classes, as variáveis argila, silte e areia muito grossa. Os centros de classes (Tabela 2) mostram que os teores mais elevados de argila estão deslocados para oeste na área de milho, de modo que a divisão em duas classes tem a Classe 1 como mais argilosa e a Classe 2 com maiores teores de areia grossa (Fig. 2A). A divisão em 3 e 4 classes mantém esse mesmo padrão na ordem das classes (Fig. 2B e C).

As Figuras $3 \mathrm{~A}$ e B mostram as subáreas de cultivo de milho variando a expressão produtiva nos diferentes anos agrícolas, para direções de semeadura diferentes. Para o primeiro ano (Fig. 3A) ocorre relativa coincidência entre as regiões de maior produtividade e a classe de maior teor de argila (Fig. 2A). Para o segundo ano (Fig. 3B) o desenho do padrão de distribuição da produção foi diferente e que pode ter sido influenciado pela mudança da direção de semeadura ocorrida neste ano, sugerindo influência do armazenamento e distribuição superficial e subsuperfical da água, além da distribuição em linha do

Tabela 2. Centro de classes (“clusters”) para as variáveis de solo mais importantes na segmentação da área experimental

\begin{tabular}{|c|c|c|c|c|c|c|c|c|c|}
\hline \multirow{3}{*}{ Atributos } & \multicolumn{9}{|c|}{ Centro de Classes (“Clusters") } \\
\hline & \multicolumn{2}{|c|}{2 Classes } & \multicolumn{3}{|c|}{3 Classes } & \multicolumn{4}{|c|}{4 Classes } \\
\hline & 1 & 2 & 1 & 2 & 3 & 1 & 2 & 3 & 4 \\
\hline Argila (\%) & 71,13 & 62,07 & 78,56 & 66,34 & 57,72 & 78,78 & 68,61 & 63,35 & 55,44 \\
\hline Silte $(\%)$ & 19,37 & 27,64 & 10,26 & 24,07 & 31,51 & 10,22 & 22,41 & 26,35 & 33,89 \\
\hline Areia muito grossa $(\%)$ & 1,88 & 2,14 & 2,06 & 1,97 & 2,96 & 2,07 & 1,84 & 2,16 & 2,20 \\
\hline
\end{tabular}


A.
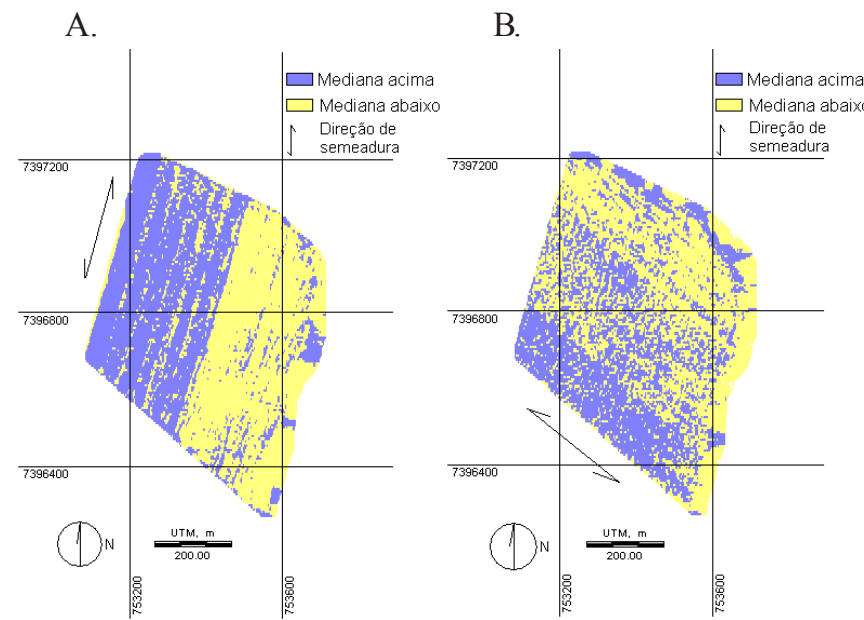

Figura 3. Distribuição dos dados de produtividade em dois quantis, tendo-se como centro a mediana $\left(6 \mathrm{t} \mathrm{ha}^{-1}\right)$, obtidos na área experimental na safra de 1999/2000 (A) e 2000/2001 (B)

adubo, como fatores condicionantes da dinâmica dos padrões de distribuição da malha de dados de produtividade do milho. Isto dá indícios de que o manejo anual pode influenciar na dinâmica da construção de zonas de manejo. Do mesmo modo, este fato sugere que a presença física do milho (raízes e parte aérea) se sobrepõe às propriedades hidráulicas do solo, tal como mostrado por Timlin et al. (2001), para a cultura da soja.

A Figura 4 mostra a segmentação feita sobre mil dados de produtividade, tomados aleatoriamente sobre o mapa da safra 1999/2000. Nota-se coerência entre o processo de segmentação multivariada e o padrão de distribuição da malha de produtividade observado na Figura 3A, já que os valores de produtividade decrescem a partir da primeira classe de agrupamento (Figuras 4A e B).

O balanço da água no solo, para as duas estações de crescimento (Figura 5A e B), revelou que não houve períodos de déficit hídrico acentuado em nenhum dos casos. Provavel-

A.

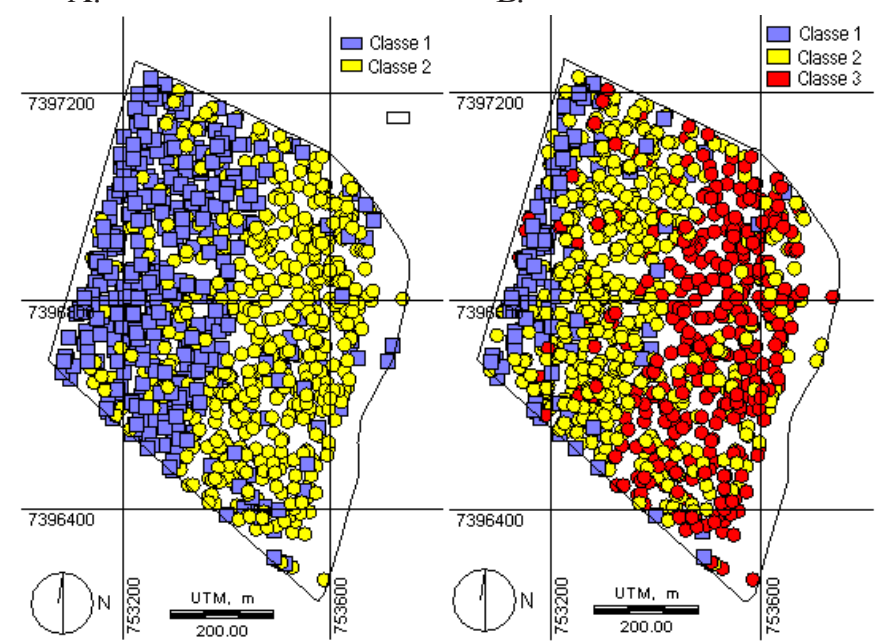

Figura 4. Distribuição de classes para os dados de produtividade obtidos na área experimental na safra de 1999/2000(A) e 2000/ 2001 (B). Para a segmentação, 1.000 dados pontuais foram tomados aleatoriamente, a partir do conjunto de 16.000 obtidos na área mente em condições hídricas favoráveis, o cotejo entre a malha de dados de produtividade e o desenho de zonas de manejo foi influenciado pelo abrandamento de estresses fisiológicos, ligados à dinâmica da água. Neste aspecto, Fridgen et al. (2000) mostraram relações diferentes para condições hídricas também diferentes. No presente caso, em que ocorrerem mais excessos hídricos que déficits, ao longo das safras é possível que tenha havido tendência para a diminuição na variabilidade dos dados de produtividade. Para se obter comprovação deste raciocínio, um número maior de anos de observação contrastantes no balanço hídrico seria necessário.
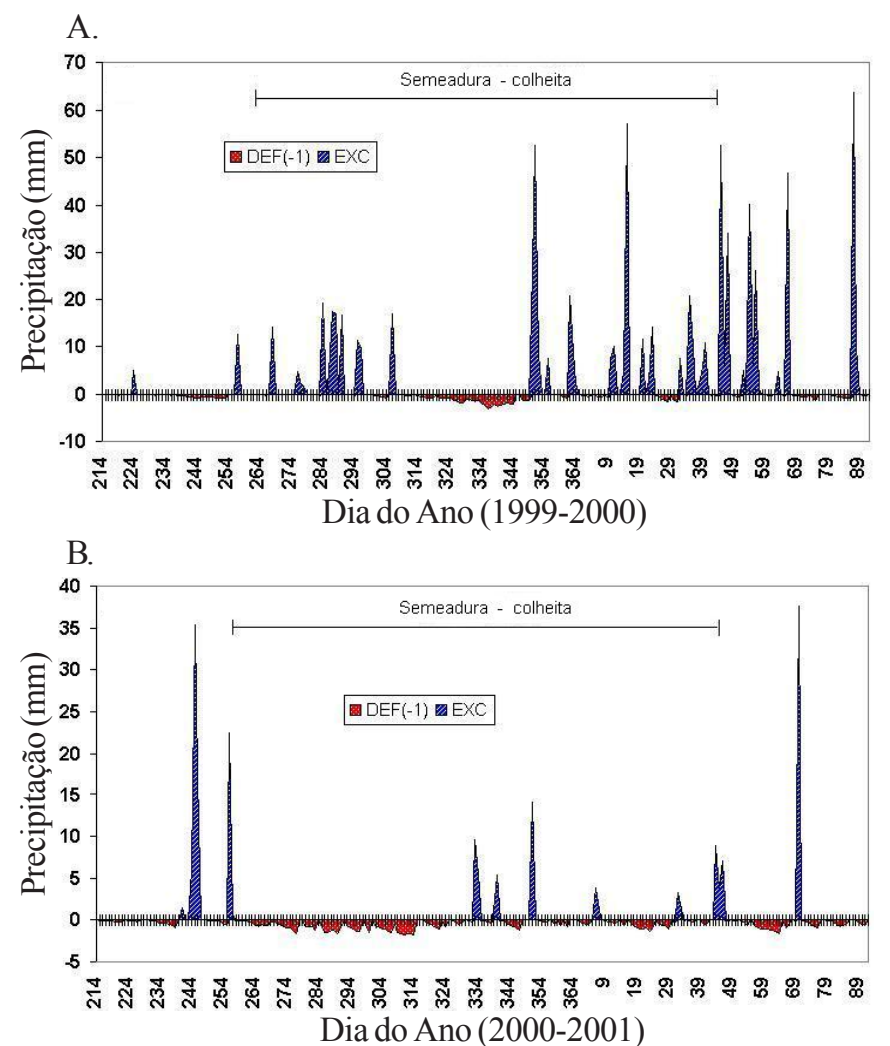

Figura 5. Extratos do balanço da água no solo para o período da semeadura à colheita, em duas safras de milho, obtidos na área experimental de 40 ha, na safra de 1999/2000 (A) e 2000/ 2001 (B)

Sendo a produtividade agrícola um fenômeno complexo sob influência de estresses múltiplos, o conjunto de variáveis utilizadas para a segmentação da área em zonas potenciais de manejo está mais ligado ao estudo da metodologia que aos fenômenos biofísicos, condicionantes da produtividade nos dois anos de observação. No entanto, as 11 variáveis de solo utilizadas estão fortemente ligadas à retenção e movimentação da água no solo, o que explica a relativa coincidência entre padrões espaciais de produtividade e a divisão em duas classes, para a primeira estação de crescimento do milho.

A análise da variância sobre o mapa de produtividade (Figuras 3A e B), considerando-se a divisão em número crescente de classes (Figura 6), mostrou que não existe ganho significativo na homogeneização da variância intraclasse, a partir de duas classes para o primeiro ano e de três classes para o segundo ano, levando-se em conta um patamar mínimo de 
$50 \%$ de queda na variância intraclasse. Tal patamar foi adotado como parâmetro expedito, abaixo do qual o aumento no número de zonas pode não ter significado prático; muito embora este índice careça de maior refinamento, outros estudos poderão aprofundar este detalhe metodológico.

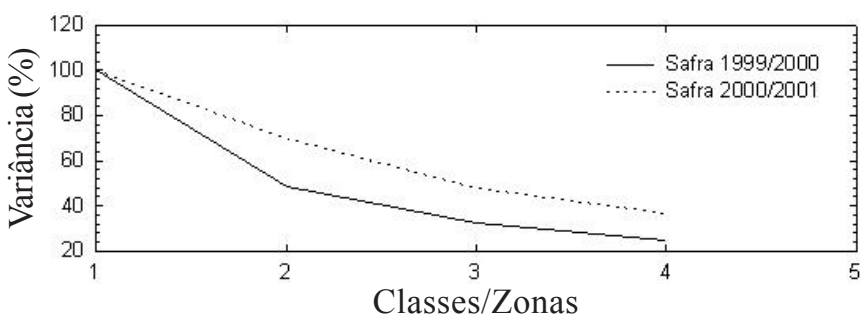

Figura 6. Modificação na variância total obtida em mapa de produtividade de duas safras de milho, para uma divisão crescente em zonas potenciais de manejo

Portanto, sendo a produtividade a expressão máxima de todos os efeitos que a condicionam em um meio, então a divisão da área em zonas de manejo seria diferente nas duas safras, sendo de 2 e 3 zonas, para o primeiro e segundo anos, respectivamente.

\section{CONCLUSÕES}

1. A integração metodológica utilizada para separar zonas de manejo mostrou-se adequada para o reconhecimento de padrões de agrupamento nas propriedades do solo.

2. O delineamento de zonas de manejo é dinâmico, podendo ser influenciado pelo manejo anual do milho, pelas variáveis utilizadas para a segmentação e pelo regime microclimático.

3. Para o milho, mudança na direção das linhas de plantio pode alterar o número e a localização das zonas de manejo.

\section{LITERATURA CITADA}

Ateca, M.R.; Sereno, R.; Apezteguía, H. Zonificacion de una superficie cultivada com soja segun aspectos fenométricos y consumo de agua del suelo. Revista Brasileira de Agrometeorologia, Santa Maria, v.9, n.1, p.111-116, 2001.

Bezdek, J.C. Pattern recognition with fuzzy objective function algorithms, New York: Plenum Press, 1981.378p.

Camargo, O.A.; Moniz, A.C.; Jorge, J.A.; Valadares, J.M.A.S. Métodos de análise química, mineralógica e física de solos do IAC. Campinas: IAC, 1986. 94p. Boletim Técnico, 106

Eastman, J.R. Idrisi for Windows: User's guide. Worcester: Clark University, 1995. 440p.

Fleming, K.L.; Westfall, D.G.; Bausch, W.C. Evaluating management zone technology and grid soil sampling for variable rate nitrogen application. In: International Conference on Precision Agriculture, 5, 2000, Bloomington. Proceedings... Madison: ASA-CSSA-SSSA, 2000. CD-Rom
Fridgen, J.J.; Kitchen, N.R.; Sudduth, K.A. Variability of soil and landscape attributes within sub-field management zones. In: International Conference on Precision Agriculture, 5, 2000, Bloomington. Proceedings... Madison: ASA-CSSA-SSSA, 2000. CD-Rom

Grimm, L.G.; Yarnold, P.R. Reading and understanding multivariate statistics. Washington, DC: APA, 2000. 371p.

Kachigan, S.K. Multivariate statistical analysis. New York: Radius Press, 1991. 303p.

McBratney, A.B.; Odeh, I.O.A. Application of fuzzy sets in soil science: fuzzy logic, fuzzy measurements and fuzzy decisions. Geoderma, Amsterdam, v.77, p.85-113, 1985.

Minasny, B.; McBratney, A.B. FuzME version 3.0. Sydney: University of Sydney, 2002. Australian Center for Precision Agriculture

Plant, R.E. Site-specific management: the application of information technology to crop production. Computers and Electronics in Agriculture, Amsterdam, v.30, p.9-29, 2001.

Raij, B.V.; Cantarella, H.; Quaggio, J.A.; Furlani, A.M.C. Recomendações de adubação e calagem para o Estado de São Paulo. Campinas: Instituto Agronômico, 1997. 285p. Boletim Técnico, 100

Santos, A.O.; Maziero, V.G.; Cavalli, A.C.; Valeriano, M.M.; Moraes, J.F.L.; Yanai, K. Monitoramento localizado da produtividade de milho cultivado sob irrigação. Revista Brasileira de Engenharia Agrícola e Ambiental, Campina Grande, v.5, n.1, p.88-95, 2001a.

Santos, A.O.; Valeriano, M.M.; Maziero, J.V.G.; Cavalli, A.C. Opportunities and limitations for the application of simulation and modeling as a support for precision farming. Acta Scientiarum, Maringá, v.23, n.6, p.1509-1517, 2001 b.

Schmidt, J.P.; Dejoia, R.K.; Young, R.K. Practical implications from variable nitrogen studies in irrigated corn. In: Robert, P.C.; Rust, R.H.; Larson, W.E. (eds.). International Conference on Precision Agriculture, 5. Proceedings... Madison, ASA, CSSA, SSSA, 2000. CD-Rom

Stewart, C.M.; McBratney, A.B. Development of a methodology for the variable-rate application of fertilizer in irrigated cotton fields. In: Robert, P.C.; Rust, R.H.; Larson, W.E. (eds.). International Conference on Precision Agriculture, 5, Proceedings... Madison, ASA,CSSA, SSSA, 2000. CD-Rom

Timlin, D.; Pachepsky, Y.; Reddy, V. R. Soil water dynamics in row and interrow positions in soybean (Glycine max L.). Plant and Soil, Dordrecht, v.237, p.25-35, 2001.

Windham, M.P. 1982. Cluster validity c-means for fuzzy clustering algorithm. IEEE Transactions. Pattern Analyses Machine Intelligence, New York, v.4, n.4, p.354-363. 1982.

Xie, X.L.; Beni, G. 1991. A validity measure for fuzzy clustering. IEEE Transactions. Pattern Analyses Machine Intelligence, New York, v.13, n.8, p.841-847. 1991. 